\title{
ESTADO DEL ARTE DE LA INVESTIGACIÓN EN LA RELACIÓN SALUD-NIÑEZ, DESDE LA PSICOLOGÍA EN RELACIÓN CON LA MEDICINA, EN BOGOTÁ
}

\author{
Ana Mercedes Abreo O*
}

\section{RESUMEN}

Se presenta el estado del conocimiento sobre salud-niñez-componente psicológico y su relación en medicina y psicología (psicoanálisis, humanismo, conductismo, sistémico), en Bogotá, durante la década del 90. La metodología es un estado del arte de tipo analítico, corte mixto, teniendo como estrategias el análisis estadístico y de contenido que se desarrolló en las fases de contextualización, compilación de evidencias, clasificación, análisis, discusión de resultados y socialización.

La poca investigación que se encuentra en contextos universitarios es discontinua y versa sobre temáticas originadas en el interés del investigador; no hay conceptualización explícita sobre salud, niñez, psique, ni avances disciplinares al respecto. La conceptualización estuvo referida a las temáticas estudiadas centradas en la enfermedad, su evaluación y tratamiento, donde el niño es el receptor del ambiente, influido por éste y, en ocasiones, víctima del mismo. El foco de la relación

\footnotetext{
Esta investigación fue realizada por la Facultad de Psicología de la Universidad Santo Tomás. Investigadores principales: Mercedes Abreo y Miryam Gastelbondo, quien acompañó el proceso hasta la fase de análisis estadístico. Auxiliares de investigación: Marcela E. Acero L. Adriana del Pilar Urueña y Mardie Millán, con el estado del arte desde la medicina alopática; Claudia Salgado con el estado del arte desde el psicoanálisis; María del Pilar Ramírez Aponte con el estado del arte desde la psicología conductual, y Luz Estella García Díaz y Sandra Rocío Ramos con las fases de compilación de evidencias, clasificación y análisis estadístico desde el humanismo rogeriano. Asesor metodológico: William Mantilla Cárdenas.Correo electrónico: muskuin@yahoo.es
} 
entre las categorías estuvo representado por la emoción como principal componente psicológico y por la incidencia del ambiente familiar sobre el niño. Desde la medicina, la psicología y dentro de los enfoques psicológicos, se encuentran puntos de convergencia y divergencia en cuanto a la comprensión de salud, niñez y su relación.

\section{Palabras claves}

Salud, niñez, psicología, medicina, estado del arte.

\section{ABSTRACT}

This article introduces knowledge state about psychological health-childhood component and its relation in medicine and psychology (psychoanalysis, humanism, behaviorism, systematic), in Bogota, 90's. methodology is a state of art of the analytical type, mixed cut, having as strategies the statistical analysis and of content developed in six phases: placing into context, evidence collection, classification, analysis, result discussion and socialization. There has been little research in university context. It is not continuous and about themes originated in the researcher's interest. There is not any explicit conceptualization about health, childhood, psychics, or disciplinary advances in this aspect. The conceptualization referred to studied themes, focused on diseases, their evaluation and treatment, where the child is the one receiving the environment, influenced by it and in occasions, its victim. The relationship between categories had as focus the emotion as main psychological component and innocence of the family environment upon the child. As from medicine, psychology and within the psychological approaches, there are convergence and divergence points as to understanding health, childhood and its relation.

\section{Key words}

Health, childhood, psychology, medicine, state of art.

\section{Introducción}

La presente investigación tuvo como pregunta guía icuál es el estado del arte de la investigación en la relación salud-niñez en el contexto de la psicología y con referencia a la medicina en la década del noventa (1990-1999), disponible en las bibliotecas de Bogotá-Colombia?

Sus objetivos estuvieron referidos a conocer cómo se ha conceptualizado y comprendido la relación salud-niñez-psique desde la medicina alopática y desde la psicología en sus diversos enfoques. Para esto, fue necesario identificar la concepción de lo psicológico, de la salud, del niño o de la niñez en cada estudio analizado, estableciendo sus convergencias y divergencias hasta hallar la relación posible.

La relación salud-niñez-componente psicológico, sobre la que esta investigación centró su atención, integra el conocimiento de, como mínimo, dos disciplinas, la medicina y la psicología, que se distinguen y complementan desde las ciencias de la naturaleza y ciencias sociales, lo cual invita a profundizar en el saber que cada una ofrece desde la investigación y a establecer -en caso que no estén dados- los vínculos o vacíos del conoci- 
miento que allí se encuentren. Por lo tanto, se trata de constituir un panorama que permita avanzar y dar continuidad a la investigación en el campo de la salud infantil, mediante la realización de "un Estado de Arte" acumulado sobre la investigación en salud-niñez-componente psicológico disponible en las bibliotecas de Bogotá y realizada durante la década del 90.

Se retomó la relación salud-niñez-componente psicológico, debido a que los tres son constituyentes del proceso de evolución de la vida y del hombre, cada uno con sus particularidades y totalidades, a modo de nodos en el entramado de interacciones que tejen su realidad, y que fue del interés de las autoras estudiar. Para las psicólogas investigadoras fue importante develar en las investigaciones fuente de estudio los conceptos allí planteados de salud, nino-niñez, psique, psicología, las posturas epistemológicas desde las cuales se abordaban dichos conceptos y la relación entre ellos.

Este estado del arte, como investigación de la investigación, permitió realizar "una investigación sobre la producción investigativa acerca de la relación salud-niñez-componente psicológico y develar la dinámica a partir de la cual se ha desarrollado la descripción, aplicación o comprensión de tal relación". Al hablar de un estado del arte, se hace referencia a la capacidad de reconocer la pluralidad de los diversos puntos de vista sobre un área del saber apoyados en diversas comprensiones del hombre; a tejer integrando, distinguiendo; lo que se constituye en un diálogo de saberes que en esta investigación se pretendió hacer al retomar la medicina alopática, el psicoanálisis, la psicología humanista rogeriana, la psicología conductual y sistémica.

\section{Concepto de psique y psicología}

Un concepto puede ser entendido como "una abstracción o idea general que puede servir de uni- dad (o de "átomo"), de teoría".... "la mayoría de los conceptos forma parte de teorías o explicaciones" (Diccionario Oxford de la Mente, 1995, p. 196); o, como plantea David Bersh (1981, p.14), "es un pensamiento acerca de las propiedades del objeto, pensamiento que usualmente es el resultado o eslabón final de una serie más o menos larga de juicios o raciocinios anteriores", enfocado a "lo que las cosas son". En todo concepto, sigue planteando Bersh, "se debe distinguir: sus propiedades esenciales o contenido y su extensión".

Las propiedades esenciales son aquellas propiedades del objeto que lo distinguen de los demás, que lo diferencian de los objetos que le son parecidos; y la extensión es la suma o totalidad de objetos que el concepto puede abarcar (limitación, generalización, definición y clasificación). Igual que como lo plantea el Diccionario Oxford de la Mente, donde la definición da cuenta del concepto, Bersh explica que el concepto se encuentra ligado a la definición, donde esta última "es una operación lógica por medio de la cual concretamos los rasgos esenciales del objeto definido y, al mismo tiempo, lo diferenciamos de todos los objetos que le son parecidos"; donde el concepto es primero y la definición es después, abarcando el concepto la definición, y no al contrario, pero donde la definición da cuenta del concepto (p. 15).

El concepto de psique, definida por los antiguos griegos como "alma, aliento o mente", está íntimamente unido a la idea de vida, pues estar vivo es tener psique, estar animado; la palabra latina "anima", a su vez, significa psique y es además un principio de individualidad, del yo (Diccionario Oxford de la Mente, 1995).

Desde filósofos como Aristóteles, Hipócrates, Platón, tener psique es ser cuerpo natural, equipado de órganos vitales y capaz de funcionar; era estar facultado con funciones de percepción, emoción, pensamiento, movimiento, nutrición; de 
automantenerse de forma independiente (Hothersall, D., 1997, cap. 1), aspectos que pueden traducirse a procesos psicológicos conectados a la salud.

El concepto de psicología, estudio de la psyque, fue evolucionando hacia una "psicología objetiva", planteada por William James (1890), y una "psicología subjetiva", planteada por Herbert Spencer (1874) (Diccionario Oxford de la Mente, 1995, pp. 964-965).

La psicología objetiva abordó el estudio de los procesos de la emoción, sensación, pensamiento, percepción, lenguaje, aprendizaje, cuando éstos se manifestaban externamente en la conducta y la psicología subjetiva que trata estas mismas facultades como directa o indirectamente concomitantes con un ajuste visible de las relaciones internas a las externas.

Los estudios de Freud desde el psicoanálisis se estaban gestando hacia 1886 desde el planteamiento clínico de "la cura mediante el habla" de los procesos subjetivos ligados al inconsciente (Hothersall, D., 1997, cap. 8).

Esta evolución en la psicología fue influida por el conductismo que limitó su definición a la psicología objetiva, excluyendo la psicología subjetiva, que luego tomó fuerza con los planteamientos de Husserl (Diccionario Oxford de la Mente, $\mathrm{p}$. 965), y dan pie al desarrollo de la psicología rogeriana.

Este breve recorrido por el concepto de psyque y de psicología presenta inicialmente un objeto de estudio claramente definido, el alma-la mente, que va asumiendo diferentes formas, dividiendo la psicología en diversas posturas ${ }^{1}$ frente a la psique -como conducta, inconsciente, el self, hasta la época actual con las pautas y patrones inte- raccionales- que resultan ser válidas, aunque no sean compartidas por todos. Válidas en cuanto que son intentos por organizar, describir y comprender la psique del hombre, aunque pareciera que el objeto de estudio no fuera el mismo; por ejemplo, el inconsciente, la conducta observable, los procesos neuropsicofisiológicos, el yo, la conciencia, la percepción, las posiciones existenciales, lo social, lo interaccional, entre otros, que seccionan la comprensión de psique.

Los focos por separado parecieran acceder a diferentes partes de lo humano, que de todas formas corresponden a la realidad, ofreciendo respuestas y comprensiones del hombre y de su psique, que son un todo para sí mismos, pero una parte para la comprensión total del hombre. Por esto, para las autoras de esta investigación fue importante retomar la definición de psique planteada por Edgar Morin en su texto El conocimiento del conocimiento (1986, cap. III) como el aspecto subjetivo del espíritu; espíritu que es una emergencia del cerebro y de su actividad neurológica, de su actividad mental que, al mismo tiempo, está enmarcada en una sociedad, en una cultura, donde todos estos elementos se conectan y retroactúan; conectándose así cerebro-cuerpo, espíritu, psique, subjetividad y sociedad, cultura. Este concepto es pertinente para el presente estudio por cuanto contempla las conexiones mencionadas, que se considera son parte de la multiplicidad de realidades que comprenden y en las que se comprende al hombre.

\section{Concepto de salud}

La conceptualización de salud se encuentra principalmente en el campo de conocimiento de la medicina. Se entiende la medicina como la ciencia, o campo del saber, que posee el conocimiento de la estructura de lo normal y de lo patológi-

1 Al referirinos a posturas, estamos hablando de una forma particular de abordar la psyque, llámese psicoanálisis, conductismo, humanismo rogeriano o sistémico. 
co, desde sus partes anatómica y fisiológica; y la medicina alopática es aquella que conoce la salud y la enfermedad desde la curación centrada en la eliminación del síntoma- (Arthur Jores, 1978).

El médico colombiano David Bersh (1981) define la salud como "un proceso continuo de variaciones ininterrumpidas que acompañan al fenómeno vital del hombre y cuyos efectos sobre dicho fenómeno se reflejan en el grado de éxito que este fenómeno tenga en el cumplimiento de su fin último, la preservación de la vida". Este mismo autor, retomando a Henrick Blum (1971), afirma que la salud es un fenómeno de dimensiones interconectadas entre las que se encuentran la genética, el comportamiento, el ambiente (éste comprende los componentes sociales, como el aislamiento, densidad, hacinamiento, competencia, seguridad, comodidad, los componentes físicos, los biológicos y los nutricionales) y los servicios en salud.

En Bersh, se destaca la idea de salud como un proceso, donde entiende proceso como el conjunto continuado de bases sucesivas de un fenómeno; es decir, un ajuste continuado que se da en un ritmo acompasado. De esta manera, supera la tradicional definición de salud dada por la OMS: "Salud es un estado de completo bienestar físico, mental y social y no solamente la ausencia de enfermedad". Además, realza, como una dimensión de la salud, la prestación de servicios en salud, lo que en Colombia remite a la Ley $100 \mathrm{de}$ 1993. La reforma a la Constitución Política de 1991 establece, mediante los artículos 48, 49 y 50 del capítulo 2: "De los derechos sociales, económicos y culturales", la obligatoriedad del Estado en la prestación de servicios públicos y de salud a la sociedad dentro de los parámetros de eficiencia, universalidad y solidaridad; reformándose así, a su vez, el Sistema de Seguridad Social en salud (Ley 100), que pretende brindar a la población co- lombiana garantía en sus derechos irrenunciables de los individuos y la comunidad para obtener una calidad de vida acorde con la dignidad humana, con los principios de eficiencia, universalidad, solidaridad, integridad unidad y participación (Ley 100, 1993).

El Estado colombiano asume la definición de salud dada por la OMS. Por esta razón, al parecer la Ley 100 pretende dar un cubrimiento total de la atención en salud a la población colombiana con miras a mejorar la calidad de vida, asumiendo como estrategias la promoción, fomento, prevención e intervención de la salud. Conceptualiza la salud como determinada en forma multifactorial y multisectorial en la que inciden entidades públicas y privadas que actúan sobre los factores de riesgo de orden ambiental, social, biológico, de comportamiento y de los servicios de salud; como "una construcción intencional e intencionada, es decir, política colectiva, ciudadana y que sólo es posible con la participación de toda la sociedad, incluyendo el Estado y sus relaciones con el capital y el mercado" (documento de la Secretaría Distrital de Salud, Dirección de Seguridad Social, POSS, 1996).

En el orden de ideas que esta ley presenta, se destaca el interés político, económico y legal, con el conociendo de factores psicológicos, médicos y sociales de la salud en una visión multidimensional y del hombre como ser biopsicosocial. Se deja de lado el componente científico de la salud y del ser humano dentro de ésta. Al citar la colectividad parece hacer mención a ésta como masa económica objeto de mercado sin dar relevancia a la familia, a las poblaciones como constructoras de la salud en un Estado, esto se ve claramente reflejado en las condiciones actuales de salud, donde existe un cobro obligatorio con mínima elección de los servicios y una mediana calidad en la prestación de los mismos. 


\section{Concepto de niñez}

En la búsqueda de la definición de niño o niñez, se encontró que está dada básicamente a nivel legal, centrada en los derechos del niño, donde el niño o niña es definido como "toda persona menor de 18 años, con excepción de aquellos casos en que las leyes de un país concreto establezcan la mayoría de edad". Convención sobre los derechos del niño, Unicef, 1989 en: www.unicef.org.

Referirse a la niñez en psicología, supone hablar de convergencias y divergencias en su concepción. Con frecuencia, se habla de niñez e infancia, refiriéndose a una etapa del desarrollo evolutivo del hombre que varía dependiendo de la mirada del autor, generalmente focalizada desde su perspectiva teórica, vale la pena decir conductual, psicoanalítica, psicodinámica, neurológica, entre otras; también puede estar focalizada por uno u otro de los procesos psicológicos como la cognición, memoria, aprendizaje; o puede centrarse en la concepción misma de desarrollo como un proceso continuo o discontinuo.

La concepción evolutiva del niño considera el desarrollo como un proceso acumulativo, en el que se van fortaleciendo las habilidades existentes desde el inicio de la vida del niño o como un desarrollo discontinuo que propone la emergencia de nuevas maneras de entender y responder al mundo, en períodos particulares del tiempo.

Naturalmente existe una tercera posición, la integradora. Lo mismo ocurre con las teorías del desarrollo; los llamados organicistas asumen la existencia de estructuras psicológicas en el niño que están en la base y controlan el desarrollo, y las teorías mecanicistas parten del hecho de que el niño es un sujeto pasivo a la estimulación ambiental (Berk, L. 1998).

También está la teoría ecológica de los sistemas, en la que Bronfenbrenner (1987) considera al niño desarrollándose dentro de un sistema complejo de relaciones afectadas por múltiples niveles del ambiente, desde los escenarios más cercanos como la familia y la escuela hasta los valores y programas culturales más extensos.

Las posibilidades son múltiples. En el caso específico de este estudio, se entiende al niño como un ser integral, constituido desde su dimensión orgánica, en relación con un ambiente físico interno y externo, no necesariamente pasivo, más sí receptivo e interactivo, que vivencia y experiencia el mundo e incide sobre él, dotado de pulsiones y deseos en relación con el inconsciente; que se desarrolla en etapas, no exactamente ordenadas y de carácter consecutivo, sino tal vez retroactivas, continuas y discontinuas. La niñez como un campo del conocimiento referido a un momento de la vida del hombre en el que confluye y se integra de manera dialógica y recursiva, una serie de componentes que constituyen al niño precisamente como hombre.

\section{Método}

Este estudio se realizó desde el pensamiento cualitativo en investigación, con el uso de técnicas de carácter mixto (interconexión de procedimientos cuantitativos y cualitativos).

Se asumió la modalidad de investigación documental en la categoría de investigación de la investigación, mayormente denominado estado del arte. Es un estado del arte de tipo analítico con el uso de la estadística inferencial y la técnica de análisis textual denominada análisis de contenido, desde Gutiérrez y Delgado, 1995.

\section{Categorías de análisis}

Las categorías principales del estudio referidas en Ios objetivos fueron los conceptos de salud, niñez-niño, psique. A partir de allí se seleccionaron como subcategorías para el análisis estadístico los 
lugares contactados, número de investigaciones seleccionadas, lugares en los que no se encontró investigaciones o de los que no se seleccionaron por no cumplir los criterios, lugares en los que se encontraron investigaciones y que cumplían con los criterios, tipo de institución, número de investigaciones seleccionadas por institución, años correspondientes a las investigaciones seleccionadas, población a la que fue dirigida, población fuente de estudio o actores, edades de los niños, temáticas psicológicas, temáticas en salud, procesos psicológicos abordados, nivel de intervención, tipo de investigación y diseños investigativos.

Para el análisis de contenido se retomaron las categorías principales seleccionadas a priori, y a partir de cada disciplina o enfoque se seleccionaron subcategorías a posteriori, las cuales se sistematizaron a través de las matrices llamadas categoriales y relacionales.

\section{Material documental}

Los documentos que se constituyeron en fuente de este estudio se seleccionaron con los criterios de ser investigaciones, abordar al mismo tiempo una temática en salud, la niñez y el componente psicológico, estar disponibles en bibliotecas de Bogotá, ser realizadas en la década del 90 , haber sido realizadas desde la medicina alopática o desde las posturas del psicoanálisis, psicología humanista rogeriana, conductista o sistémica.

\section{Instrumentos}

- Tabla de lugares contactados. Se realizó con el fin de poder contar con la identificación de los lugares en los que se encuentran investigaciones sobre el tema, disponibles en bibliotecas de Bogotá.

- Tabla de inventario general. Ofrece una panorámica general de los sitios visitados y de las investigaciones encontradas en cada uno.
Incluye el título de la investigación, autor, año de elaboración, fuente, número de ubicación (topográfico) y si fue seleccionada o no para el presente proyecto.

- Tabla de memorias de lugares contactados. En ella se consignó la experiencia de las investigadoras durante las fases de compilación de evidencias y clasificación; es un resumen de diario de campo que da cuenta del contacto institucional y del contexto de la fuente del trabajo seleccionado o los motivos por los que no fue seleccionado.

- Fichas descriptivas. Recopilan la información básica de cada investigación seleccionada; título de la investigación, autor(es), año, número topográfico, ubicación, objetivo general, objetivos específicos, metodología, tipo de investigación, conclusiones y aportes.

- Matriz categorial. Organiza la información del análisis de contenido. Incluye el nivel del texto (contenido sintáctico), las notas del investigador (nivel semántico) y el contexto (nivel pragmático).

- Matriz relacional. En esta matriz se logran conexiones y distinciones desde la relación entre las categorías de la matriz categorial.

\section{Procedimiento}

El procedimiento se realizó con miras a lograr la producción de un meta-texto analítico propio del análisis de contenido y propósito de este estado del arte. Incluyó las fases de contextualización, compilación de evidencias, clasificación, análisis estadístico y de contenido, discusión de resultados y socialización de resultados.

\section{Resultados}

Se contactaron 81 instituciones en sus diversas modalidades, según aparecen en la tabla 1. Los 
lugares contactados fueron clínicas y hospitales, seguido por organizaciones privadas, universidades, instituciones gubernamentales y organizaciones académicas. Los criterios para contactarlas fueron la relación con la salud infantil, el abordaje del componente psicológico, la existencia de centro de documentación y la suposición de que investigaban.

Tabla 1. Relación de lugares contactados

\begin{tabular}{|llrc|}
\hline Lugares contactados & Número & \multicolumn{1}{c|}{$\%$} \\
\hline 1 & Universidades & 16 & 19.75 \\
\hline 2 & $\begin{array}{l}\text { Organizaciones } \\
\text { gubernamentales }\end{array}$ & 15 & 18.52 \\
\hline 3 & $\begin{array}{l}\text { Organizaciones sin ánimo } \\
\text { de lucro y privadas }\end{array}$ & 18 & 22.22 \\
\hline 4 & Clínicas y hospitales & 26 & 32.10 \\
\hline 5 & Organizaciones académicas & 6 & 7.41 \\
\hline & Total & 81 & 100.00 \\
\hline
\end{tabular}

Se encontró que en las instituciones de salud, estatales o privadas, poco se investiga en la relación salud-niñez-psicología, a menos que sean hospitales universitarios o que tengan contacto directo con universidades. Muchas de estas instituciones no cuentan con centros de documentación.

Como se puede observar en el gráfico 1, de las 81 instituciones contactadas, en 58 de ellas (70.73\%) no se encontró investigación sobre el tema.

En las 23 instituciones restantes (29.10\%) se encontraron investigaciones sobre salud infantil, pero no en todas se encontraron investigaciones de acuerdo con los criterios establecidos. De esas 23 instituciones, se encontraron estudios pertinentes en 14 de ellas (17.07\%); es decir que de las 81 instituciones visitadas, sólo 14 cumplieron los criterios de abordar la relación salud-niñez-psicología, haber realizado estos estudios en la década de los 90 y tener publicado o disponible al públi- co el material mediante centros de documentación. Este grupo lo constituyeron 10 universidades, 2 hospitales, 1 institución privada y 1 institución estatal (podría también decirse que son tres las instituciones estatales, por cuanto tres de ellas -H. Militar, H. de la Misericordia y Universidad Nacional- fueron seleccionadas; sólo que por la intersección que presentan se clasificaron por su misión, en las que se encontró un total de 59 investigaciones pertinentes para este estudio.

Se seleccionaron 59 investigaciones de una población de 169 en medicina y 163 en psicología (tabla 2). De estas 59, corresponden 15 a medicina y 44 a psicología.

\section{Gráfico 1. Relación instituciones e investigación}

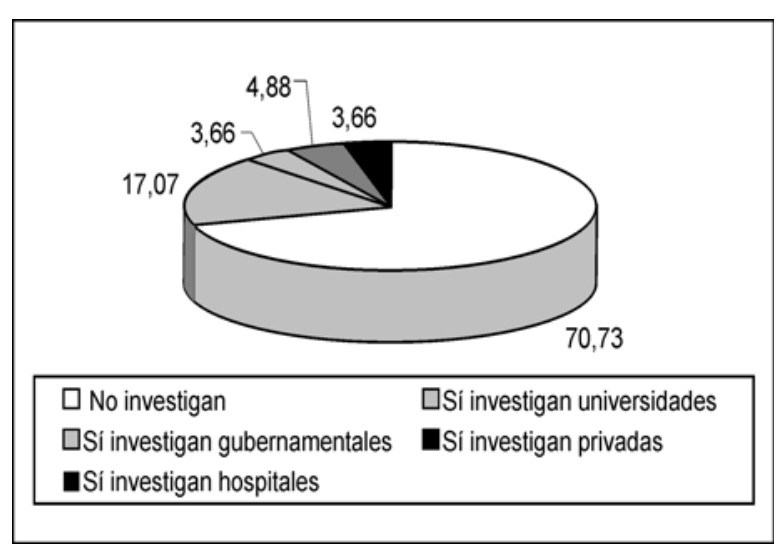

De manera más detallada, y en términos porcentuales, la institución con mayor aporte en medicina fue el Hospital de la Misericordia con un $8.47 \%$ (donde se encontraron investigaciones realizadas por estudiantes de la Facultad de Medicina de la Universidad Nacional), seguido por la Universidad del Rosario y del Bosque con un $5.08 \%$ cada una. En psicología, la institución que mayor aporte realizó a este estudio fue la Universidad Santo Tomás. De acuerdo con cada perspectiva, en humanismo la Universidad Santo Tomás obtuvo un $6.78 \%$; la Universidad Nacional en 
psicoanálisis, 8.47\%; en el enfoque conductual la Universidad de La Sabana, 6.78\%; y la Universidad Santo Tomás alcanzó un $18.64 \%$ en el enfoque sistémico.

Uno de los criterios de selección de las investigaciones y categoría de análisis en este estudio fue el año en que la investigación se realizó, década del 90 (tabla 2). Al respecto, se encontró que en 1990 se presentó el mayor número de investigaciones en la relación salud-niñez-psicología desde la medicina y desde la psicología (16.95\%), seguido por 1996 (13.56\%). Sin embargo, no se encuentran estadísticamente datos significativos, por cuanto se presenta dispersión.

Al respecto de las temáticas en salud, se encontró gran variedad, motivo por el cual no se obtuvo porcentajes al no observarse diferencias significativas entre los datos. De manera descriptiva, puede decirse que la investigación en salud infantil y componente psicológico se ha enfocado en la enfermedad caso que ocurre en la medicina, conductismo y enfoque sistémico. En psicoanálisis, la mitad de las investigaciones no refieren problemática en salud; $y$ en humanismo, a pesar de abordar enfermedades, también se hace referencia a la capacidad cognitiva-intelectual. En lo pertinente a la enfermedad, se nota una tendencia a investigar en discapacidad física, sensorial, intelectual, dándose un número de 10 investigaciones referidas a la discapacidad.

Sobre los temas psicológicos, se encontró que éstos, al igual que las temáticas en salud, no muestran diferencias estadísticamente significativas. Descriptivamente, en medicina se hace énfasis en la emoción, siendo éste el componente psicológico considerado por las investigaciones seleccionadas; y en psicología, la temática varía de acuerdo con el enfoque. El enfoque que presenta mayor continuidad en las temáticas es el sistémico, enfatizando en la cohesión, adaptabilidad y comunicación familiar. Entre los enfoques no se encuentran similitud en las temáticas, como tampoco entre la medicina y la psicología.

De los procesos psicológicos que abordan las investigaciones, el más estudiado es la emoción (38.98\%). Posteriormente, como aparece en la tabla 3, se encontró que el $32.20 \%$ de los estudios analizados no incluye ningún proceso psicológico básico.

Dentro del porcentaje del $38.98 \%$, los estudios más contribuyentes obedecen a la medicina y al conductismo, seguido por el enfoque sistémico. En el psicoanálisis, no apareció la emoción como

Tabla 2. Relación años e investigación

\begin{tabular}{cccccccc}
\hline Año & Medicina & Psicoanálisis & Conductismo & Humanismo & Sistémico & Total & $\%$ \\
\hline 1990 & 2 & 0 & 3 & 4 & 1 & 10 & 16.95 \\
1991 & 0 & 0 & 1 & 0 & 0 & 1 & 1.695 \\
1992 & 0 & 1 & 2 & 0 & 3 & 6 & 10.17 \\
1993 & 2 & 0 & 1 & 0 & 3 & 6 & 10.17 \\
1994 & 2 & 2 & 2 & 0 & 1 & 7 & 11.86 \\
1995 & 2 & 0 & 3 & 1 & 1 & 7 & 11.86 \\
1996 & 4 & 0 & 2 & 0 & 2 & 8 & 13.56 \\
1997 & 2 & 1 & 0 & 1 & 3 & 7 & 11.86 \\
1998 & 1 & 0 & 0 & 0 & 0 & 1 & 1.695 \\
1999 & 0 & 2 & 3 & 0 & 1 & 6 & 10.17 \\
Total & 15 & 6 & 17 & 6 & 15 & 59 & 100 \\
\hline
\end{tabular}


Tabla 3. Procesos psicológicos

\begin{tabular}{|l|c|c|c|c|c|c|c|}
\hline PROCESO & Medicina & Psicoan. & Conductismo & Humanis & Sistém & Suma & $\%$ \\
\hline Emoción & 9 & 0 & 9 & 1 & 4 & 23 & 38.98 \\
\hline Percepción & 0 & 0 & 1 & 2 & 0 & 3 & 5.08 \\
\hline Atención & 1 & 0 & 0 & 0 & 0 & 1 & 1.69 \\
\hline Memoria & 0 & 0 & 0 & 0 & 0 & 0 & 0.00 \\
\hline Lenguaje & 0 & 0 & 1 & 0 & 0 & 1 & 1.69 \\
\hline Aprendizaje & 3 & 0 & 4 & 1 & 0 & 8 & 13.56 \\
\hline Motivación & 0 & 0 & 0 & 0 & 0 & 0 & 0.00 \\
\hline Pensamiento & 0 & 0 & 1 & 2 & 0 & 3 & 5.08 \\
\hline Varios & 0 & 0 & 0 & 0 & 1 & 1 & 1.69 \\
\hline Ninguno & 2 & 6 & 1 & 0 & 10 & 19 & 32.20 \\
\hline Total & 15 & 6 & 17 & 6 & 15 & 59 & 100 \\
\hline
\end{tabular}

proceso fundamental, se trabajan factores emocionales no entendidos explícitamente como procesos psicológicos. El enfoque humanista presenta un predominio por los procesos cognitivos.

En el gráfico siguiente se muestra la cantidad y el porcentaje del tipo de intervención. El tipo de intervención que se encontró más utilizado fue la evaluación (45.76\%), seguido por el tratamiento (32.2\%). En medicina se encontró predominio de la evaluación; en psicoanálisis tiende a no especificarse, seguido por la intervención; en humanismo, al igual que en el psicoanálisis, tiende a no especificarse seguido por la evaluación; en conductismo se da un marcado predominio en intervención; y en sistémico, en evaluación.

Gráfico 2. Tipo de intervención

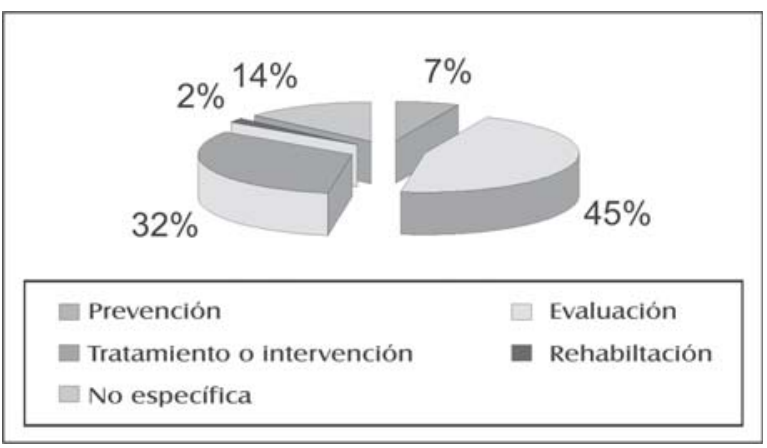

Con respecto al tipo de población con la que se realizan los estudios (gráfico 3), éstos son niñas y niños $(57.63 \%)$, seguido por el abordaje de las familias (20.34\%). El número de las familias se encuentra relacionado con los estudios desde la perspectiva sistémica y humanista principalmente. Los estudios no citan distinciones entre la población de niños y niñas, según el género. Las disciplinas y los enfoques no mostraron diferencias, podría decirse que el sistémico aborda en prioridad la familia, aunque hay que tener en cuenta que estas investigaciones abordan la familia con inclusión del niño.

\section{Gráfico 3. Población fuente de estudio $o$ actores}

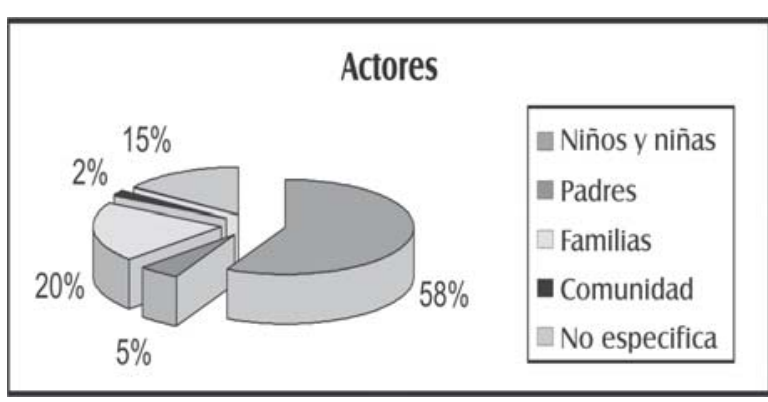

Las edades de los niños se sistematizó por rangos, debido a que cada investigación presentaba edades diversas, algunas sin una especificación clara de las mismas; de tal manera que se escogieron los rangos que más se aproximaban a los trabajos analizados (tabla 4). 
Tabla 4. Franjas de edad

\begin{tabular}{|lccccccc|}
\hline RANGO DE EDADES & Medicina & Psa & Conduct. & Humanismo Sistémico & Total & $\%$ \\
\hline NEONATOS & 1 & 0 & 0 & 0 & 1 & 2 & 3.39 \\
\hline De 1 mes a 5 años & 2 & 0 & 2 & 0 & 0 & 4 & 6.78 \\
\hline De 6 a 10 años & 3 & 2 & 1 & 0 & 1 & 7 & 11.86 \\
\hline De 1 mes a 10 años & 0 & 0 & 4 & 0 & 2 & 6 & 10.17 \\
\hline De 11 a 15 años & 2 & 0 & 3 & 0 & 0 & 5 & 8.475 \\
\hline De 1 mes a 15 años & 3 & 0 & 4 & 0 & 4 & 11 & 18.64 \\
\hline De 16 a 20 años & 0 & 0 & 0 & 0 & 0 & 0 & 0 \\
\hline De 1 mes a 20 años & 1 & 0 & 0 & 0 & 2 & 3 & 5.085 \\
\hline No especifica edad & 3 & 4 & 3 & 6 & 5 & 21 & 35.59 \\
\hline Total & 15 & 6 & 17 & 6 & 15 & 59 & 100 \\
\hline
\end{tabular}

Tabla 5. Población a la que se dirige la investigación

\begin{tabular}{|lccccccc|}
\hline Población & Medicina & \multicolumn{2}{l}{ PSA Conductismo Humanismo Sistémico } & Total & $\%$ \\
\hline Comunidad & 0 & 0 & 0 & 0 & 1 & 1 & 1.695 \\
\hline Profesionales de la salud & 11 & 6 & 14 & 4 & 13 & 48 & 81.36 \\
\hline Profesores de la educación & 0 & 0 & 3 & 2 & 0 & 5 & 8.475 \\
\hline Familias & 0 & 0 & 0 & 0 & 1 & 1 & 1.695 \\
\hline Padres & 3 & 0 & 0 & 0 & 0 & 3 & 5.085 \\
\hline Niños & 0 & 0 & 0 & 0 & 0 & 0 & 0 \\
\hline No especifica & 1 & 0 & 0 & 0 & 0 & 1 & 1.695 \\
\hline Total & 15 & 6 & 17 & 6 & 15 & 59 & 100 \\
\hline
\end{tabular}

Se observa que el mayor porcentaje corresponde a la "no especificación" de la edad (35.59\%); la edad más estudiada fue el rango comprendido entre el primer mes de nacido y los 15 años (18.64\%), dentro del cual se incluyen los rangos de 6 a 10 años (11.86\%) y el rango de 1 mes a 10 años (10.17\%).

En conclusión, puede decirse que los niños entre el primer mes de nacimiento y los 15 años son los más abordados en las investigaciones, franja de edad que coincidió con la elección previa de las investigadoras de este estado del arte (0-12 años), rango que fue ampliado para el análisis estadístico, por cuanto las investigaciones abordadas así lo exigieron.

De acuerdo con la tabla 5, se puede identificar que la mayoría de las investigaciones va dirigida a los profesionales de la salud (81.36\%). En mínima proporción, los trabajos se dirigen a la comunidad en general o a las familias y, como dato 
Tabla 6. Tipo de investigación

\begin{tabular}{|lcccccccc|}
\hline TIpo & Mediclna & PSA & Conductlsmo & Humanlsmo & SIstémIco & Total & $\%$ \\
\hline Cuantitativo & 3 & 0 & 12 & 0 & 3 & 18 & 30.51 \\
\hline Cualitativo & 1 & 1 & 5 & 2 & 7 & 16 & 27.12 \\
\hline Mixta & 0 & 0 & 0 & 0 & 1 & 1 & 1.695 \\
\hline No especifica & 11 & 5 & 0 & 4 & 4 & 24 & 40.68 \\
\hline Total & 15 & 6 & 17 & 6 & 15 & 59 & 100 \\
\hline
\end{tabular}

muy importante, en ninguna proporción a los niños o niñas.

Respecto al tipo de investigación, el mayor porcentaje corresponde a la subcategoría no especifica (40.68\%); lo que significa que, a pesar de que las investigaciones dan cuenta del método, poco lo hacen discriminando el tipo de investigación. La tabla 6 muestra que el tipo de investigación más utilizado es el cuantitativo (30.51\%) de predominio para el conductismo; la investigación cualitativa es retomada en su mayoría por la visión sistémica.

La metodología de las investigaciones varía notablemente de acuerdo con el enfoque y disciplina. A pesar de la diversidad, podría decirse que el método más utilizado en la investigación en salud infantil en medicina alopática y psicología es el descriptivo (18.64\%), como aparece en la tabla 7, seguido por el método experimental propio de la medicina y el conductismo (11.86\%). En la especificación por disciplina y enfoque, se encuentra que en medicina el más utilizado es el método descriptivo; en psicoanálisis no se especifica; en humanismo, el fenomenológico; en conductismo, el método experimental; $y$ en sistémico, el comparativo.

Sobre los conceptos de salud, niñez y psique, abordados desde el análisis de contenido, se encontró que explícitamente los trabajos no presenta- ron conceptualización sobre estas categorías ni definiciones. Al hacer el análisis de contenido en medicina, se devela que el niño, en cuanto hombre, es concebido como un ser biopsicosocialmultidimensional y que la salud se ve afectada por múltiples factores de tipo biológico, psicológico, económico, legal y social, que son retomados y analizados por los médicos al abordar y explicar las alteraciones de la salud, sólo que al momento de la intervención se centran en el componente biológico desde la terapia farmacológica, incluyendo la psiquiatría.

Los médicos reconocen la niñez como una etapa o un momento en la vida del ser humano, que está en constante desarrollo y, por lo tanto, necesita del apoyo y afecto de un grupo familiar estable, en especial de la madre. El desarrollo del niño, al igual que la salud, puede ser influenciado por múltiples factores, destacándose los socioeconómicos, que posibilitan el acceso a la educación, a una alimentación determinada, a servicios públicos indispensables y a la recreación, entre otros. Desde la incidencia económica y también legal, identifican a un niño, desde su mayoría de edad, 18 años o hasta los 21, por la dependencia económica y social.

El componente psicológico es comprendido como la emoción o el comportamiento, especialmente la emoción, reconociendo que en el niño existe un desarrollo psicológico que depende en gran 
Tabla 7. Metodología utilizada en las investigaciones

\begin{tabular}{|lccccccc|}
\hline Metodología & Med. & PSA & Conduct. & Hum. Sistémico & Total & \% \\
\hline Cuasi-experimental & 1 & 0 & 2 & 0 & 1 & 4 & 6.78 \\
\hline Preexperimental & 0 & 0 & 1 & 0 & 0 & 1 & 1.695 \\
\hline Experimental & 0 & 0 & 7 & 0 & 0 & 7 & 11.86 \\
\hline Técnico experimental intrasujeto & 0 & 0 & 1 & 0 & 0 & 1 & 1.695 \\
\hline Línea de base de tipo intrasujeto & 0 & 0 & 2 & 0 & 0 & 2 & 3.39 \\
\hline Longitudinal & 0 & 0 & 2 & 0 & 0 & 2 & 3.39 \\
\hline Retrospectivo & 1 & 0 & 0 & 0 & 0 & 1 & 1.695 \\
\hline Exploratorio, prospectivo, descriptivo & 1 & 0 & 0 & 0 & 0 & 1 & 1.695 \\
\hline Estudio piloto de corte & 1 & 0 & 0 & 0 & 0 & 1 & 1.695 \\
\hline Revisión documental & 2 & 1 & 0 & 1 & 1 & 5 & 8.475 \\
\hline Descriptivo & 3 & 0 & 1 & 1 & 6 & 11 & 18.64 \\
\hline Comparativo & 1 & 0 & 1 & 0 & 2 & 4 & 6.78 \\
\hline Observación participante & 0 & 0 & 0 & 0 & 1 & 1 & 1.695 \\
\hline Inductivo expost-facto & 0 & 0 & 0 & 1 & 1 & 2 & 3.39 \\
\hline De cohorte histórico y descriptivo & 1 & 0 & 0 & 0 & 0 & 1 & 1.695 \\
\hline Piloto epidemiológico & 1 & 0 & 0 & 0 & 0 & 1 & 1.695 \\
\hline Fenomenológico & 0 & 0 & 0 & 3 & 0 & 3 & 5.085 \\
\hline No especifica & 3 & 5 & 0 & 0 & 3 & 11 & 18.64 \\
\hline Total & 15 & 6 & 17 & 6 & 15 & 59 & 100 \\
\hline
\end{tabular}

medida del contexto en donde se desenvuelve, particularmente la familia y el contacto madre-hijo.

Desde la psicología y sus diversas posturas conceptuales, se encontró que con respecto a la salud en el psicoanálisıs no existe una línea divisoria, una diferencia tajante entre la salud y la enfermedad, entre salud mental y trastorno mental, como sí existe en la medicina; no puede existir una división entre la salud corporal, física y salud mental. En todos los seres humanos, plantea el psicoanálisis, se presentan elementos patológicos, por sanos que puedan parecer y viceversa; en la persona más patológica, más alterada desde el punto de vista mental, existirán aspectos sanos. Es importante conectar aquí con el concepto de salud de D. Bersh (1981), como un proceso continuo desde la concepción hasta la muerte, encontrándose la coexistencia de salud y enfermedad, donde la una incluye a la otra más que excluirla. Esto en relación con una de las conceptualizaciones encontradas a nivel teórico del psicoanálisis sobre salud, donde se entiende la salud como un proceso: "el proceso de salud ha de ser entendido como una capacidad de vivir de forma autónoma, solidaria y con capacidad de alegría, gozo. En donde salud, tanto individual como social, serán unos componentes psicológi- 
cos y unos sociales. Es decir que la salud será un problema y un concepto ecológico, pues afecta y es afectado por el conjunto de los subsistemas del ecosistema humano" (Ferrero, J. 1983, referenciado por Salgado, C. 2001, p. 60).

Desde el psicoanálisis, entonces, pareciera considerase la salud como algo que no es estable, como no lo es tampoco el trastorno, ni la enfermedad. Al parecer, según los trabajos estudiados, no puede existir una división entre la salud corporal, física y salud mental. Según esta postura conceptual, una enfermedad altera siempre las capacidades psicológicas y las formas de relacionarse. Donde las emociones o afectividad humana significativa para el sujeto conlleva cambios relevantes en el cuerpo, ya que de alguna manera los síntomas físicos de una enfermedad aumentan o disminuyen según el estado afectivo y emocional del individuo. Se entienden los síntomas físicos como un recurso protector de escape que refleja los conflictos internos inconscientes y permite excusarse de ellos.

En lo referente a la niñez, no se habla de niñez, sino de infancia, en la cual se definen estados biológicos -etapas psicosexuales-, por los que el sujeto debe atravesar para un buen desarrollo de su personalidad; aspecto que se destaca en el pensamiento freudiano (Ferrero, J., 1983, referenciado por Salgado, C., 2001). Igualmente, se habla de niño como un sujeto que vive ciertas experiencias psíquicas que le ayudan a definirse en cuanto a su opción sexual y a formarse como un sujeto que tiene un lugar específico en el mundo y para quien es de trascendental importancia la relación establecida con la madre, entendiéndose madre no necesariamente como la madre biológica, sino como esa figura materna que le ofrece protección, afecto y cuidados. Se deja ver al niño como un sujeto de deseo, que atravesado y determinado por el deseo constituye su psique y determina su existir en el medio, en su interacción con el otro; esto más con rela- ción a la postura lacaniana, según la cual "el sujeto es siempre alguien que busca algo perdido, que es él mismo: su referente de identidad, el sujeto es aquel capaz de tener un deseo y un objeto del deseo. La madre es el objeto primordial de los deseos..." (Salgado, C. 2001, p. 52).

En la concepción de lo psicológico, el psicoanálisis no trabaja procesos psicológicos básicos como memoria, pensamiento, lenguaje, cognición, ni cómo se produce la psique, sino en cómo funciona el inconsciente. De esta forma, concibe la psique como un compuesto de fuerzas o cargas energéticas que generalmente están opuestas entre sí. Estas fuerzas inconscientes son el id, el ego y el superego.

Pero además de estas fuerzas inconscientes, la psique también se constituye en estructuras, como el inconsciente, preconsciente y consciente.

Desde el humanismo rogeriano, la salud es vista como una interrupción del proceso evolutivo del hombre, como un evento tensionante que genera aislamiento, frustración, depresión y otras obstrucciones en el crecimiento personal del niño. Este enfoque centra el tratamiento y terapia en un proceso de aprendizaje cuyo objetivo es que el hombre -niño- vivencie, experimente, nuevas posibilidades que le permitan, superando la enfermedad, crecer y fortalecer el sí mismo. Si esto sucede, la enfermedad puede convertirse en un factor potencializador para el desarrollo humano. La enfermedad vista así no se da por sí sola, sino por la incidencia de procesos interventivos. Estos estudios destacan la postura humanista rogeriana donde "la tendencia del ser humano es hacia el pleno desarrollo físico y mental, que requiere de condiciones adecuadas y la ausencia de obstáculos para llegar a ser armónico y feliz" (Martínez, M., 1988).

La niñez es entendida como etapas del desarrollo humano dividida en primera y segunda infancia 
de acuerdo con lo planteado por la psicología evolutiva, donde el niño es un ser social, existencial, que se encamina hacia la autonomía, pero que, en su condición de menor evolución, se considera pasivo-receptivo con respecto al adulto, con menores capacidades cognoscitivas y de comprensión del mundo, donde el desarrollo del sí mismo está ligado al ambiente que lo rodea, determinado principalmente por sus padres desde incluso antes de nacer, donde su existencia obedece, en primer lugar, a las expectativas de los padres, a lo que se espera de él en el futuro; se considera tan determinado por el medio que, en muchos casos, "víctima".

La concepción de psique se comprende desde el sí mismo. Los procesos psicológicos que se destacan son, en primera instancia, la emoción, posteriormente la cognición y el aprendizaje. La emoción se encuentra ligada a la expresión de sentimientos que, a su vez, se relaciona con la posibilidad de bienestar del individuo, donde en la medida en que el niño logre tener una mejor y mayor identificación de sus sentimientos propios podrá expresarlos pertinentemente y esto influirá en su condición de estar sano o saludable. En este mismo sentido, la cognición, como proceso en el cual se encuentra la construcción del esquema corporal y concepto de sí mismo, favorece el desarrollo del niño como ser autónomo y social, con capacidad de toma de decisiones, que se construirá en la medida en que pueda tener opciones de aprendizaje.

Desde la perspectiva conductual, la salud es entendida desde la medicina, posiblemente por su conexión a esta disciplina desde la medicina conductual, en la que se incorporan elementos teóricos y metodológicos propios de la terapia y modificación del comportamiento y las aplica al campo de la salud y la enfermedad (Morales Calatayud, F. referenciado por Ramírez, p. 45). Todos los estudios abordados retoman una enfermedad, dándole relevancia y entendiéndola como una afección a la constitución biológica del organismo que genera cambios conductuales y que también puede ser producida por cambios conductuales; en otros estudios, la enfermedad es entendida como una amenaza o dolor.

Conciben al niño como un ser biopsicosocial que está en constante interacción con el medio, que a la vez se ve influenciado por los padres, especialmente por la madre y por personas con quien interactúa habitualmente, así como también por los factores socioeconómicos que lo rodean. La niñez es vista como una etapa de la vida del ser humano en la que el niño está en constante desarrollo y requiere del apoyo y afecto de la familia y de todos los seres que conforman su entorno, en donde en los casos de enfermedad depende del afecto materno. Durante la enfermedad, el desarrollo del niño también puede verse influido por factores socioeconómicos que actúan como causales de modificaciones psicológicas, de tal modo que la niñez y la salud van de la mano. Así mismo, esta psicología rescata la importancia de resaltar la estabilidad emocional tanto del niño como de la familia y la comprensión del niño como ser que necesita afecto y apoyo de su familia, lo cual contribuye al mantenimiento de su salud. La visión que le da importancia al aspecto emocional permite inferir que lo psicológico se comprende en momentos como lo emocional o lo conductual.

La psique es entendida como la conducta; la conducta, como la manifestación observable o perceptible de las fuerzas que motivan la acción humana. El estudio de la conducta está enmarcado dentro de la comprensión positivista.

Desde la psicología sistémica, tampoco se hace referencia a la salud, sino a la enfermedad. Ésta es entendida básicamente como un estresor que genera desestabilidad en el sistema familiar como contexto en el cual se encuentra inmerso el niño. La enfermedad como estresor conlleva a la movi- 
lización de procesos de ajuste y adaptación frente a lo cual la familia habrá de reorganizarse haciendo uso de sus recursos y dinámica familiar, de tal manera que el estresor, generador de crisis en el sistema familiar, puede llevar al estancamiento o regeneración de la familia.

La niñez está entendida como parte del proceso evolutivo del hombre, más específicamente con los estadios de su ciclo vital, que se encuentra a su vez conectado con el ciclo vital del sistema familiar. El niño, por su parte, es visto, al igual que el hombre, como un sistema abierto, sólo que, a diferencia del hombre adulto, es entendido como influenciado por el adulto, considerado en una posición de desventaja que lo posiciona en términos de poder como víctima en la interacción.

Al parecer, el componente psicológico es entendido como los vínculos afectivos y comunicacionales que se construyen en la interacción con otros. Los procesos psicológicos no son abordados como tal, más si se hace referencia a ellos al retomar el vínculo afectivo-emocional en la cohesión familiar y en la comunicación formadora de pautas de interacción. Un proceso que apareció con gran importancia en las investigaciones retomadas fueron los de ajuste y adaptación, procesos relevantes para la psicología, por cuanto marcan comprensiones sobre la interacción del hombre con su medio.

El paradigma que se encontró como base en estas conceptualizaciones fue el psositivismo, el cual se observó presente en el conocimiento de la medicina alopática y del conductismo. No podría decirse a qué tipo de positivismo obedece, por cuanto no fue objetivo de este estudio una puntualización minuciosa al respecto; lo que sí puede decirse es que en estos trabajos el hombre y la realidad son vistos desde el control, la causalidad, medición, cuantificación, referentes observables, con la intención de describir, explicar y predecir las acciones humanas.
Desde el psicoanálisis no pudo identificarse un paradigma guía, más aún teniendo en cuenta que Ios trabajos eran retomados desde Freud y Lacan. Sin embargo, y sin ánimo de considerar estos estudios como positivistas, se hacía referencia a un determinismo psíquico en la vida del niño, que puede entenderse desde las relaciones causales, lineales.

Fue interesante encontrar que desde los trabajos del humanismo rogeriano y sistémico -que escapan aparentemente al paradigma positivista y se enmarcan desde el existencialismo-fenomenología y paradigma sistémico respectivamente-, se encontró una mezcla paradigmática donde un sujeto observa a un objeto convertido en muestra en el ámbito metodológico. Estos trabajos presentaban teorías y planteamientos teóricos propios a sus respectivos enfoques y posturas paradigmáticos, con interpretaciones por parte de los autores del estudio de tipo determinista lineal.

En cuanto a la relación entre salud-niñez-componente psicológico, se encontró que en medicina y en psicología la salud se encuentra influenciada por factores emocionales, siendo así mismo la emoción y/o vinculación afectiva-emocional el proceso psicológico que prima en las investigaciones, e incluso es considerada como el componente psicológico en estudio. Es común también para las dos disciplinas el puntuar desde la enfermedad más que desde la salud, donde se encuentra aún en esta década la concepción dual de salud- enfermedad. La enfermedad en un niño se encuentra conectada con su vinculación emocional con su contexto más cercano, la familia, de la que la madre es la figura principal, al estar mayormente conectado con él.

Otra relación se da en el ámbito de la causalidad y la multicausalidad. La salud se considera en los estudios abordados desde las dos disciplinas como influenciada por múltiples componentes o dimensiones, entre ellos: genéticos, bioquímicos, 
nutricionales, ambientales, comportamentales, etc. Esta relación obedece posiblemente a la concepción de la salud y del hombre como biopsicosociales.

Junto con la comprensión de la relación salud-niñez-psicología, esta investigación tuvo la intención de encontrar los puntos de encuentro $y$ desencuentros entre la medicina y la psicología no sólo a nivel cualitativo -mediante el análisis estadístico-, sino también desde el análisis de contenido. Al respecto, se encontraron más encuentros que desencuentros.

Como puntos de encuentros se tiene que:

- En cuanto a la conceptualización de salud, se encuentra marcada la dualidad salud-enfermedad. Donde la enfermedad está connotada negativamente como amenaza, estresor y disrupción de la vida, de la organización de Ios sistemas, del crecimiento personal. Este punto coincide con el planteamiento de Manrique, E., 1994 (referenciado por Salgado, 2001, p.82), cuando dice que "la definición de la salud que desde siempre ha caracterizado la conceptualización y las prácticas médicas y ha conducido a que la investigación en el campo de la salud esté fundamentalmente dominada por el afán del avance en el saber no propiamente de la salud, sino específicamente de la enfermedad. De esta forma, el ser humano se ha movilizado en su afán o, más bien, preocupación por combatir la enfermedad", en consonancia con Bersh, D. (1981), cuando afirma que «Es muy difícil desplazar las nociones tradicionales de salud y enfermedad... Así, todo el tiempo se establece una dicotomía entre estos dos términos que se asocian diariamente, convirtiéndose así los problemas de salud en problemas de enfermedad".

- El niño es concebido desde el nivel evolutivo y como ser social en desarrollo, con caracte- rísticas diferentes a las del adulto; determinado o influenciado por el ambiente del cual él es receptor generalmente pasivo donde en muchas ocasiones termina siendo víctima del adulto.

- La emoción se rescata como el componente psicológico principal, por lo que aparte de la conducta y la interacción puede considerarse como la manifestación psíquica relevante a nivel infantil.

Los puntos de desencuentros se presentan básicamente desde el psicoanálisis y desde humanismo rogeriano en relación con las otras posturas.

- Tanto el psicoanáilisis como el humanismo rogeriano retoman investigaciones que no necesariamente abordan enfermedad, aún manteniendo la dualidad salud-enfermedad, se puntúa también desde un sujeto o niño sano.

- Desde el psicoanálsis, el niño está determinado por la relación vincular con la madre y el padre; sin embargo, no como sujeto pasivo, sino como activo desde su propio deseo. Esta perspectiva es la única que plantea como posibilidad interventiva la oferta de la escucha, diferente a las demandas frecuentes de intervenir para procesos de adaptación. La escucha de un discurso dentro de un espacio en donde la palabra se libere y se abra paso al diálogo como facilitador de una intervención integral que involucre al niño, sus padres y la institución. Escuchar la voz del niño.

\section{Discusión y conclusiones}

Esta investigación deja ver tres aspectos centrales: 1. El tipo de investigación encontrada y el avance en el conocimiento disciplinar al respecto. 2. La discontinuidad en la investigación y el distanciamiento en la relación ciencia, educación superior, problemática social 3. La concepción de niño. 
- La investigación encontrada es de tipo formativo, por cuanto hace parte de la actividad académica del profesional en formación donde el objetivo primordial va dirigido al desarrollo de competencias investigativas en el estudiante y cuyo resultado es el ejercicio investigativo. Estos ejercicios investigativos, tal como se encontraron en este estado del arte, responden al tipo de preguntas investigativas que parten del interés y experiencia personal del investigador, en este caso el estudiante, en ocasiones conectada a su práctica profesional y que se encamina al conocimiento de una temática en particular dentro de su campo disciplinar de formación, y no a preguntas de conocimiento disciplinar dentro de la investigación innovativa.

Al parecer, Ios profesionales, una vez egresados, dedican sus esfuerzos e intereses al servicio asistencial de la población infantil. De los 59 estudios abordados, 5 estudios en medicina y los 14 estudios sobre psicología sistémica de la USTA correspondieron al desarrollo conceptual de preguntas investigativas sugeridas por investigadores con experiencia (Md. Rafael Vásquez y la Ps. Ángela Hernández) y que implicaron continuidad en el conocimiento.

- El que la investigación se haya encontrado a cargo de las universidades, de manera discontinua, y en el dominio del interés de los estudiantes tiene una doble implicación. Por un lado, destaca el importante papel de la responsabilidad social de las universidades, su compromiso con la sociedad; de otro lado, la discontinuidad refleja una vez más el distanciamiento del conocimiento generado en estas instituciones con la realidad social. Como lo afirma Gordillo 2004, "la presencia entre los contenidos educativos de todos los niveles de numerosos conocimientos y des- trezas científicas y tecnológicas no se ha articulado tradicionalmente con acercamientos que planteen su contextualización social". "La presentación educativa de la ciencia y la tecnología ha estado dominada, más bien, por formatos en los que los conocimientos científicos parecen hallazgos o descubrimientos al margen de condicionantes históricos e ideológicos, como heroicos logros de genios individuales". Por lo tanto, como lo continúan diciendo estos autores en sus respectivos escritos, se requiere de la presencia activa, creativa y constructiva de las universidades a través de la conexión ciencia y realidad social, superando el interés aislado del investigador, sin querer decir por esto que tal aspecto no sea importante; por lo que se hace necesario articular propuestas concretas y articuladas con la sociedad.

Así mismo, expresa Acevedo (2004): “El desarrollo de la tecnociencia contemporánea plantea a la humanidad innumerables desafíos. Por su parte, los estudios de contexto, en la investigación social sobre ciencia y tecnología, exigen abordar las relaciones universidad-sociedad, partiendo de un enfoque claramente diferenciado, máxime cuando se trata de un país con graves conflictos como Colombia".

- Como se presentó en los resultados, el paradigma que marcó la investigación en la relación estudiada fue el positivista; éste ha tenido presencia en la ciencia, no sólo en la psicología, sino también en la medicina. Entiéndase paradigma en el sentido de Moliner (1984, referenciado por Vallés, 1999) como "Tratado de los métodos del conocimiento científico, en general o de determinada ciencia" (p. 47); paradigma que marca la concepción fenomenológica, ontológica y metodológica en la ciencia. Sin ánimo de enjui- 
ciar al positivismo como afirma Gordillo 2004, actualmente se requiere de "una ruptura definitiva con la concepción positivista de las relaciones entre ciencia y sociedad que fue dominante durante los dos primeros tercios del siglo XX".

Esa concepción positivista que se denota en la mayoría de investigaciones y simplista de la realidad y del hombre -en este caso del niño-, como refiere Morin en su texto de Introducción al pensamiento complejo, ubica al niño en una relación sujeto-objeto de conocimiento. Donde el niño visto en posición inferior a la del adulto, incompleto en su concepción (por no estar completo en su desarrollo evolutivo en relación con el adulto) se convierte en objeto de otro, ya sea la familia, la sociedad o las leyes que necesitan existir para protegerlo de ese otro, o del investigador mismo, donde no hace parte activa del inicio de la investigación, no más allá del referente que el adulto considera necesario. Es necesario preguntarse hasta qué punto esta visión del niño hace parte de un principio dormitivo en el que sociedad-conocimiento conforman una díada que contribuye a mantener problemáticas en torno a la infancia, como el maltrato infantil.

\section{BIBLIOGRAFÍA}

ACEVEDO P, Elsa Beatriz (2004). Relaciones entre universidad y sociedad en los estudios sociales de ciencia y tecnología en Colombia. En: http:// www.oei.org/ctsi

BERSH, David. El fenómeno de la salud. Bogotá: OPS, OMS/ ASCOFAME, 1981.

BERK, Laura. Desarrollo del niño y del adolescente. España. Ed. PrenticeHall, 1998.
BOGOTÁ Secretaría Distrital de Salud. Dirección de Seguridad Social. División de Prestación de Servicios en Salud. Documentos sobre Plan Obligatorio de Salud Subsidiado. Santafé de Bogotá, 1996.

BRONFENBRENNER, U. La ecología del desarrollo humano: experimentos en entornos naturales $y$ diseñados. Barcelona: Paidós, 1987.

GORDILLO, Mariano Martín y LOPEZ, José A. (2004). Acercando la ciencia a la sociedad: la perspectiva CTS su implantación educativa. En: http:// www.oei.org/ctsi

GUTIÉRREZ, Juan y DELGADO, Juan Manuel. Métodos y técnicas de investigación en ciencias sociales. Madrid: Ed. Síntesis S.A., 1995.

HOTHERSALL, David. Historia de la psicología. México: McGraw-Hill, 1997.

JORES, Arthur. La medicina en la crisis de nuestro tiempo. México: Ed. Siglo Veintiuno, 1978.

MANTILLA CÁRDENAS, William. La ciencia y el científico. Bogotá: UNAD. Ed. Guadalupe, 1995.

MARTíNEZ, Miguel. La psicología humanista. MéxiCO: Ed. Trillas, 1998.

MORIN, Edgar. El método. El conocimiento del conocimiento. Ed. Cátedra, 1986.

RAMÍREZ, María del Pilar (2003). Estado del arte de la investigaciín en la relación salud-niñez-psique desde la psicología conductual, realizada en la década del noventa y disponible en bibliotecas de Bogotá-Colombia. Trabajo de grado para optar al título de psicóloga. Bogotá: Universidad Santo Tomás.

REPÚBLICA DE COLOMBIA. Constitución Política de Colombia. Santafé de Bogotá, 1991. 
REPÚBLICA DE COLOMBIA. Sistema de Seguridad Social. Ley 100 de 1993. Santafé de Bogotá, 1993.

SALGADO, Claudia. Estado del arte de la investigación en la relación salud-niñez-psique desde el psicoanálisis, realizada en la década del noventa y disponible en bibliotecas de Bogotá-Colombia. Trabajo de grado para optar al título de psicóloga. Bogotá: Universidad Santo Tomás, 2001.
SINGUENZA PINILLA, C. Diccionario Oxford de la Mente. Madrid: Edición Richard L. Gregory. Alianza, 1995.

VALLÉS, Miguel. Técnicas cualitativas de investigación Social. Madrid: Ed. Síntesis, 1999.

UNICEF (1989). Convención sobre los derechos del niño. En: www.unicef.org. 